Western University Scholarship@Western

Research Summaries

BrainsCAN

2018

\title{
The effects of habits on motor skill learning
}

BrainsCAN, Western University

Nicola J. Popp

Western University

Atsushi Yokoi

Western University

Paul Gribble

Western University

Jörn Diedrichsen

Western University

Follow this and additional works at: https://ir.lib.uwo.ca/brainscanresearchsummaries

Part of the Neurosciences Commons

\section{Recommended Citation}

BrainsCAN, "The effects of habits on motor skill learning" (2018). Research Summaries. 1.

https://ir.lib.uwo.ca/brainscanresearchsummaries/1

This Book is brought to you for free and open access by the BrainsCAN at Scholarship@Western. It has been accepted for inclusion in Research

Summaries by an authorized administrator of Scholarship@Western. For more information, please contact tadam@uwo.ca, wlswadmin@uwo.ca. 


\section{The effects of habits on motor skill learning}

\section{Background}

Humans are capable of astonishing feats of motor performance, such as performing athletics, playing music and dancing. But what does it take to become an expert? Are thousands of hours of practice enough for anyone to achieve a skilled performance? Or to what degree are the training and instructions you receive important in the acquisition of motor expertise?

When practicing a novel skill, our movements are quite variable in the beginning. Over time, they become more accurate and consistent. This indicates that a 'motor habit' is forming, the actions no longer require as much conscious thought and effort (known as 'automatized' movements) which decreases the 'cognitive demand' placed on our brain to complete movements and actions.

However, habits are sometimes rigid and can also hinder performance. For instance, the motor habit we develop when typing out words on a keyboard can interfere with typing on a foreign keyboard on which symbols are mapped differently onto keys. So while habits play an integral part in motor skill learning, preventing bad habits from forming and reinforcing good ones is a key objective when teaching novel motor skills.

While highly relevant in everyday life, there has been little experimental research examining the formation and persistence of motor habits.

\section{The Research}

Chunking is a natural phenomenon that we use to break up long sequences of items or actions to aid memorization. For instance, a phone number is chunked in a way that makes it easy to remember: area code - prefix - line number.

We used chunking as a tool to study how subjects learned a specific series of movements over the course of three weeks and how habits formed in that learning process.

\begin{abstract}
Key Points
Learning new motor skills can involve adopting motor habits that could help or hinder overall performance outcomes. Which habit(s) a participant adopt(s) can be induced early on through instruction. These motor habits can be rigid and persistent during motor skill training and practice but it is also possible to influence or change motor habits through training.
\end{abstract}

\section{Publication}

Neurology.org October 2017

bit.ly/NIPO0518-R

\section{BrainsCAN Support}

Human Cognition and Sensorimotor Core Imaging Core

\section{Research Support \\ JSMF, NSERC, CFREF, CIHR, NICHD \\ Western Faculty, Group or Institution}

Brain and Mind Institute

Participants typed long sequences of numbers as quickly and accurately as possible on a keyboard-like device. Early in their training we instructed them to memorize the sequences using chunking arrangements we supplied. These supplied arrangements would either be helpful in performing the task quickly or hinder a participant's speed, as follows.

We had already established in a separate experiment that participants could transition between two adjacent fingers (i.e. pressing the first finger then the second finger) quicker than they could press the same finger twice (i.e. the first finger followed by the first finger again). Since the individual chunks were only two or three digits long and therefore not challenging memory, we considered this to be a biomechanical constraint. 


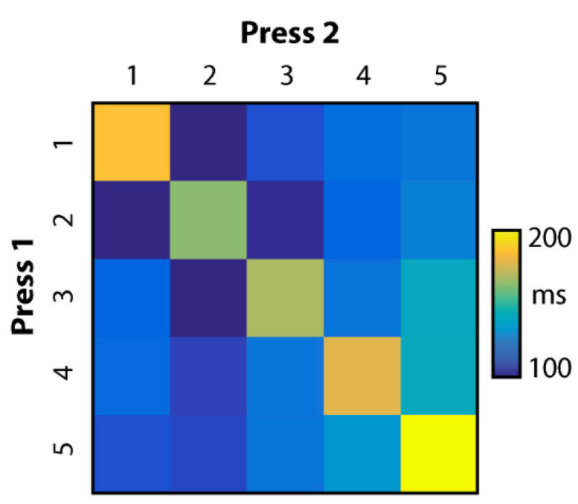

Figure 1: Delays between pairs of keypresses

We also consistently saw that the delay between chunks was longer than between key presses within a single chunk - for the pattern 321 123, the delay between 321 and 123 was consistently longer than the delay between individual numbers within a chunk, 3 then 2 then 1 . Since we had already determined the delay between finger presses within a single chunk was a biomechanical constraint, the longer delay between chunks suggests that a participant is using some extra time to recall the next chunk from memory.

We used these two findings to create 'helpful' or 'unhelpful' chunking patterns - by placing the break for two chunks between adjacent finger presses, we proposed to insert the memory recall delay into a transition that would otherwise be quick, slowing the performance of the whole sequence overall (say, ...32 112 33...); conversely, by placing the break for two chunks between repeated finger presses, we proposed that the slower repeated finger movement could happen at the same time as the recall of the next chunk from memory, benefitting overall performance (compare the earlier chunking pattern to ...321 123 3...). Adopting the helpful pattern would be a good motor habit, adopting the unhelpful one would be bad - we determined which chunking pattern was provided.

We studied how our initial instructions led to the formations of these motor habits and whether participants were able to change these habits with training.

\section{The Findings}

We were able to induce lasting habits based on our initial instructions. For most participants, the beneficial habits created a long-term performance benefit and bad habits prevented them from achieving the highest level of performance. This shows that instructions early in training can impact performance over a prolonged period of time. We also found that some participants were able to modify the induced habits and find novel ways of chunking the sequences that led to improved performance.

Our findings support the idea that motor skills are critically impacted by the initial training and instructions received.

\section{Next Steps}

Now that we have developed a way to induce and study habits in motor skill learning, we can investigate how to help people overcome bad motor habits and how to reinforce good habits. Is there a critical period for habit formation? What are the most efficient techniques to avoid the formation of bad habits? Many important questions concerning habits that directly relate to our everyday life and training in such domains as sports and music are still unanswered.

Our findings provide novel insights into the interaction of cognition, habits and motor optimization.

\section{Western Researchers}

Nicola J. Popp

Atsushi Yokoi

Figure credits

Figure 1 - BioRxiv, the authors
Paul Gribble

Jörn Diedrichsen 